\title{
Gene Encoding a c-Type Cyclin in Mycosphaerella graminicola Is Involved in Aerial Mycelium Formation, Filamentous Growth, Hyphal Swelling, Melanin Biosynthesis, Stress Response, and Pathogenicity
}

\author{
Yoon-E Choi and Stephen B. Goodwin \\ United States Department of Agriculture-Agricultural Research Service, 915 West State Street, Department of Botany and \\ Plant Pathology, Purdue University, West Lafayette, IN 47907-2054, U.S.A.
}

Submitted 21 April 2010. Accepted 14 December 2010.

\begin{abstract}
Mycosphaerella graminicola is an important wheat pathogen causing Septoria tritici blotch. To date, an efficient strategy to control M. graminicola has not been developed. More significantly, we have a limited understanding of the molecular mechanisms of $M$. graminicola pathogenicity. In this study, we attempted to characterize an $M C C 1$-encoding c-type cyclin, a gene homologous to FCC1 in Fusarium verticillioides. Four independent $M C C 1$ knock-out mutants were generated via Agrobacterium tumefaciens-mediated transformation. All of the MCC1 mutants showed consistent multiple phenotypes. Significant reductions in radial growth on potato dextrose agar (PDA) were observed in all of the MCC1 mutants. In addition, MCC1 gene-deletion mutants produced less aerial mycelium on PDA, showed delayed filamentous growth, had unusual hyphal swellings, produced more melanin, showed an increase in their stress tolerance response, and were reduced significantly in pathogenicity. These results indicate that the $M C C 1$ gene is involved in multiple signaling pathways, including those involved in pathogenicity in M. graminicola.
\end{abstract}

The ascomycete Mycosphaerella graminicola (anamorph Septoria tritici) is one of the most important pathogens on wheat, causing a serious foliar disease known as Septoria tritici blotch (STB). This disease has been reported in most wheat-growing regions around the world, including the United States (Polley and Thomas 1991). When environmental conditions are favorable for the growth of $M$. graminicola, STB can cause significant yield loss of wheat. In spite of the importance

Corresponding author: S. Goodwin; E-mail: sgoodwin@purdue.edu and Steve.Goodwin@ARS.USDA.gov

Mention of trade names or commercial products in this publication is solely for the purpose of providing specific information and does not imply recommendation or endorsement by the United States Department of Agriculture.

* The $\boldsymbol{e}$-Xtra logo stands for "electronic extra" and indicates that two supplementary figures and one supplementary table are published online and that Figures 1, 2, 3, and 4 appear in color online.

This article is in the public domain and not copyrightable. It may be freely reprinted with customary crediting of the source. The American Phytopathological Society, 2011. of STB, strategies for efficient management of $M$. graminicola have not been developed. Currently, breeding for resistant cultivars and treatments with fungicides are used to manage STB. However, neither of these methods provides complete control of STB due to rapid evolution of $M$. graminicola populations and frequent sexual recombination. New haplotypes with resistance to fungicides and that can overcome resistant cultivars arise rapidly. Furthermore, recent concerns over environmental issues can restrict fungicide applications and limit the number of compounds available. Therefore, it is necessary to better understand $M$. graminicola biology which, in turn, will help to establish an efficient strategy to manage STB.

Cyclins are a highly conserved family of proteins which are involved in cell cycle progressions via the activation of cyclindependent protein kinases (CDK). There are several types of cyclins which control different parts of cell cycles through activation of appropriate CDK partners. Among these cyclins, ctype cyclin is distantly related to the others and is unique in that it is involved in transcriptional regulation (Kuchin et al. 1995; Liao et al. 1995). In Saccharomyces cerevisiae, the c-type cyclin protein Ume $3 p$ acts as a repressor of gene transcription. In response to appropriate environmental cues such as stress conditions, Ume $3 p$ is destroyed at the protein level via ubiquitin-mediated proteolysis. After Ume3p is degraded, the expression of many downstream genes is initiated at the transcriptional level (Cohen et al. 2003; Cooper et al. 1997). Recently, an emerging body of information indicates that c-type cyclin in filamentous fungi is implicated in many different signaling pathways, including fungal development, toxin metabolism, and pathogenicity. In Fusarium verticillioides, the FCCl gene encoding a c-type cyclin was identified as an important regulator in the biosynthesis of the mycotoxin fumonisin as well as the asexual development of microconidia (Shim and Woloshuk 2001). In F. graminearum, the FCC1-homologous gene CIDI is involved in conidiation, vegetative growth, pigmentation, and pathogenicity (Zhou et al. 2010). Therefore, it is obvious that c-type cyclins in F. verticillioides and $F$. graminearum are involved in multiple important signaling pathways. However, to our knowledge, except for FCC1 and CID1, no other c-type cyclin gene has been reported in filamentous fungi.

To determine the roles of c-type cyclin in M. graminicola, we identified and characterized the MCCl gene that is homologous to $F$. verticillioides $F C C 1$ and $F$. graminearum $C I D 1$. As with $F C C 1$ and $C I D 1, M C C 1$ gene-deletion mutants 
showed pleiotropic effects on growth, filamentous growth rate, hyphal swelling, melanin biosynthesis, stress tolerance, and pathogenicity. Our results indicate that the $M C C 1$ gene encoding a c-type cyclin in $M$. graminicola impacts multiple signaling pathways, including those for growth, differentiation, secondary metabolism, and pathogenesis.

\section{RESULTS}

The $M C C 1$ gene encodes a c-type cyclin.

We searched for c-type cyclin genes in M. graminicola using the sequence of the $F$. verticillioides c-type cyclin, FCCI (GenBank accession number AF294431), as query. A putative homolog of FCC1 was identified in the M. graminicola genomic database. This gene was located on $M$. graminicola chromosome 9, specifically at the sequence from bases $1,594,202$ to $1,595,191$. Analysis of the identified sequence revealed that it had significant similarity to various c-type cyclin genes across different fungal species. For example, the predicted gene shares high similarity with FICl (XP_755914.1) in Aspergillus fumigatus (E value, 2e-86), CID1 (XP 384531.1 ) in $F$. graminearum (E value, 1e-76), and $F C C \overline{1}$ (AAK30047.1) in F. verticillioides (E value, 5e-76). Based on this similarity, we designated the identified c-type cyclin gene in $M$. graminicola as $M C C 1$ (probable $M$. graminicola c-type cyclin 1). Amino acid alignments of $M C C l$ with c-type cyclin
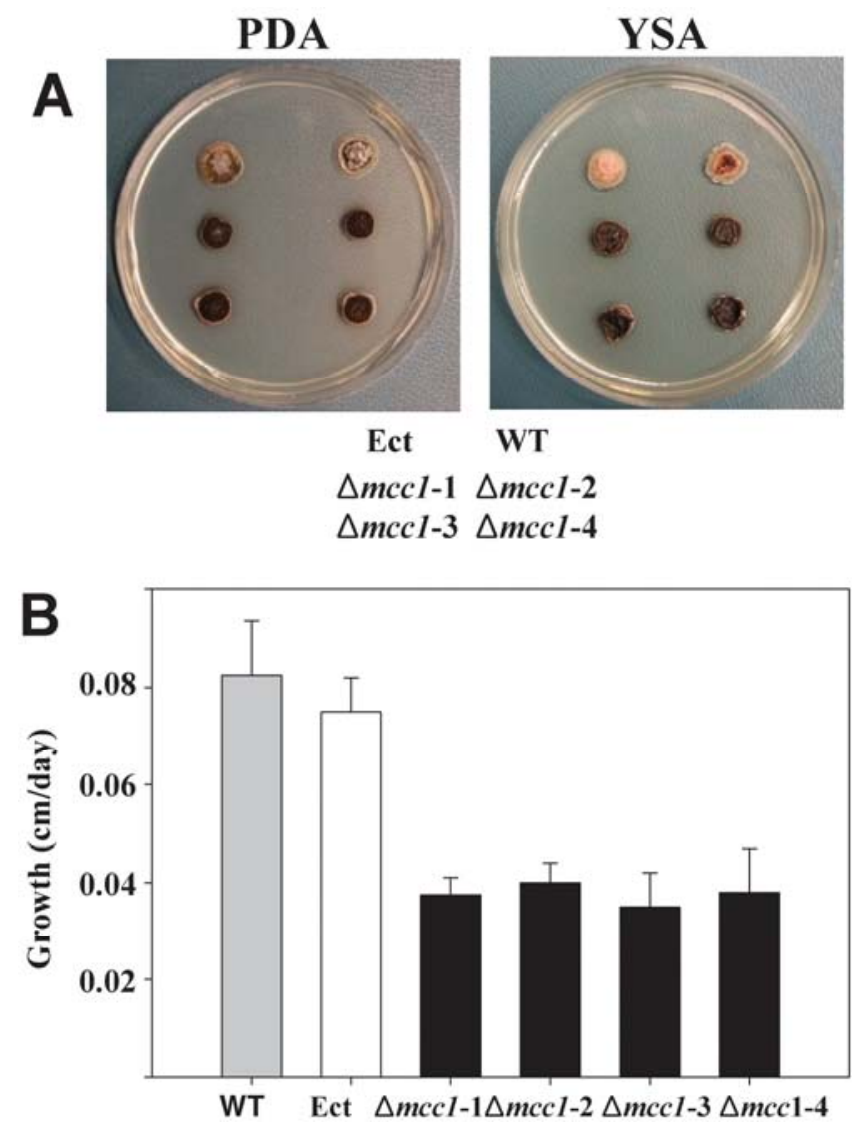

Fig. 1. Morphology and radial growth on agar plates analysis. A, Growth morphology on agar plates was tested with the wild type (WT), ectopic transformant (Ect), and four independent $M C C 1$ gene-deletion mutants. All strains were grown on potato dextrose agar (PDA) or yeast-sucrose agar (YSA) for 20 days. Growth was reduced and dark pigmentation was significantly increased in four $M C C 1$ mutant isolates ( $\triangle m c c 1-1, \Delta m c c 1-2$, $\Delta m c c 1-3$, and $\Delta m c c 1-4)$. B, Radial growth on PDA was measured as the diameter of colonies and presented as a bar graph. Three biological replications were performed. Thin lines above the bars indicate standard errors. proteins in $F$. verticillioides $(F C C 1)$ and $F$. graminearum (CIDI) indicated high similarity in the sequences (Supplementary Fig. 1). Further in silico analysis showed that the MCC1 gene encodes 327 amino acids with cyclin domains from amino acids 53 to 142 and 155 to 236 . An RXXL domain previously shown to be required for self destruction also was found from amino acids 25 to 28 .

\section{MCC1 gene-deletion mutants were generated} via Agrobacterium tumefaciens-mediated transformation.

To test the functional roles of $M C C 1$ in $M$. graminicola, we generated $M C C 1$ gene-deletion mutants by Agrobacterium tumefaciens-mediated transformation (ATMT) (Mullins et al. 2001). Among 144 hygromycin-resistant transformants, four independent MCC1 gene-deletion mutants were identified by polymerase chain reaction (PCR) screens with primers $\mathrm{MCC} 1-$ che-F1 and R1. The MCC1-che-F1 and R1 primers are located on the upstream and downstream flanking regions of the $M C C 1$ open reading frame (ORF) and the resultant $M C C 1$ gene-deletion mutants should have a gene-replacement event substituting the entire MCC1 ORF with a hygromycin phosphotransferase $(H P H)$ gene (Supplementary Fig. 2a). Based on that, we identified gene-deletion mutants of $M C C 1$ by the size of PCR reaction amplicons. The wild-type and ectopic control strains had a 1.3-kb band originating from the MCC1 ORF, whereas $M C C 1$ gene-deletion mutants had instead a 1.6-kb band from the $H P H$ gene. Transformation was confirmed with another set of primers, MCC1-neg-F1 and R1, located within the $M C C 1$ ORF. The wild-type and ectopic control strains generated a $0.7-\mathrm{kb}$ band from the $M C C 1$ ORF, whereas MCC1 gene-disruption strains did not produce detectable PCR amplicons, indicating that the gene was absent in the $M C C 1$ genedeletion mutants. We designated the four independent mutants as $\Delta m c c 1-1, \Delta m c c 1-2, \Delta m c c 1-3$, and $\Delta m c c 1-4$. We also included an isolate containing the DMC1 construct integrated elsewhere in the genome as an ectopic control and designated it "Ect".

\section{The $M C C 1$ gene-deletion mutants are defective} in growth on agar plates.

We first tested whether $M C C 1$ gene deletion can cause morphological differences on yeast-sucrose agar (YSA) or potato dextrose agar (PDA) plates. Four independent $M C C 1$ gene-disruption strains showed consistent differences in growth and produced darker pigmentation compared with the wild-type progenitor (Fig. 1A). Regardless of the incubation temperature, whether $18^{\circ} \mathrm{C}$ or room temperature, we observed the same phenotypic differences between the wild type and $\Delta m c c 1$ mutants (data not shown). We observed consistent growth reduction in the four independent MCC1 gene-deletion mutants compared with the wild-type and ectopic control strains. Measurement of the radial growth of $M$. graminicola strains on PDA plates revealed that $\Delta m c c l$ mutants grew at approximately $50 \%$ the rate of the wild-type progenitor and the ectopic control strain (Fig. 1B). From these data, we concluded that $M C C l$ in $M$. graminicola affects radial growth on agar plates in a positive manner.

\section{The $M C C 1$ gene-deletion mutants are defective} in formation of aerial mycelium on agar plates.

When we observed the growth patterns on PDA under a dissecting microscope, we noticed remarkable differences between the wild-type or ectopic control strains and $\Delta m c c l \mathrm{mu}-$ tants. The wild-type and ectopic control strains developed long aerial mycelia, whereas $\Delta m c c l$ mutants produced limited aerial mycelia (Fig. 2). These phenotypic differences appeared consistently across four independent $M C C 1$ gene-disruption mutants ( $\Delta m c c 1-1, \Delta m c c l-2, \Delta m c c l-3$, and $\Delta m c c l-4)$. The 
wild-type and ectopic control strains developed thick, white mycelia. In contrast, aerial mycelium was almost nondetectable in $\Delta m c c 1$ mutants (Fig. 2). We extracted aerial mycelium with $0.15 \%$ Tween 20 and quantified its amount with a hemacytometer. The $\Delta m c c l$ mutants produced approximately less than $10 \%$ of the aerial mycelium of the wild-type or ectopic control strains (data not shown). Our data clearly indicated that $M C C 1$ influences the formation of aerial mycelium in M. graminicola in a positive manner.

\section{$M C C 1$ gene deletion resulted in a significant increase} in melanin biosynthesis.

Deletion of the $M C C 1$ gene resulted in increased dark pigmentation compared with the wild-type progenitor, which showed a lack of dark pigment (Figs. 1A and 3A). Melanin is commonly responsible for the dark pigmentation in filamentous fungi. We followed an extraction and measurement protocol specifically designed for melanin (Babitskaya et al. 2000) to estimate the amounts of melanin produced by each strain with three biological replications on agar plates (YSA), as well as in liquid medium (yeast-sucrose broth [YSB]). We observed more than a 15-fold increase in melanin production in $\Delta m c c l$ mutants ( $\Delta m c c 1-1, \Delta m c c 1-2, \Delta m c c 1-3$, and $\Delta m c c 1-4)$ on YSA culture when compared with the wild-type progenitor or ectopic control (Fig. 3B). In addition, we also observed an approximately sevenfold increase in production of melanin in four independent $M C C l$ disruption mutants in YSB (Fig. 3B). Increased melanin production in $\Delta m c c l$ mutants in YSB was
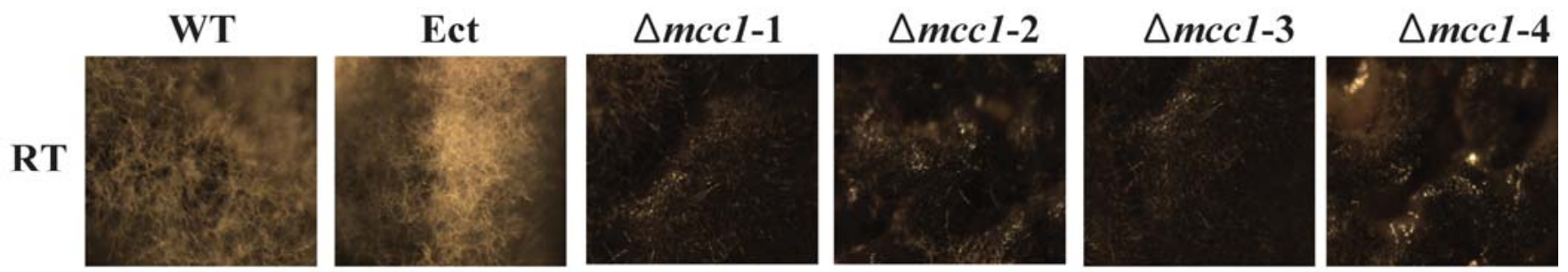

$18^{\circ} \mathrm{C}$
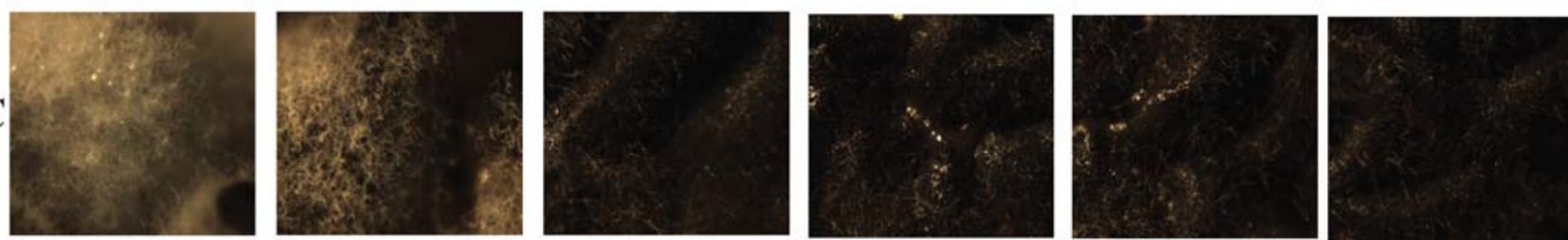

Fig. 2. Aerial mycelia in the $M C C 1$ mutants. A, Aerial mycelium was photographed with a dissecting microscope. All strains were grown on potato dextrose agar after 20 days at room temperature or at $18^{\circ} \mathrm{C}$ (indicated on the left). In the wild type (WT) and ectopic transformant (Ect), abundant aerial mycelia were present. However, aerial mycelium was rarely observed in four independent $M C C 1$ mutants.
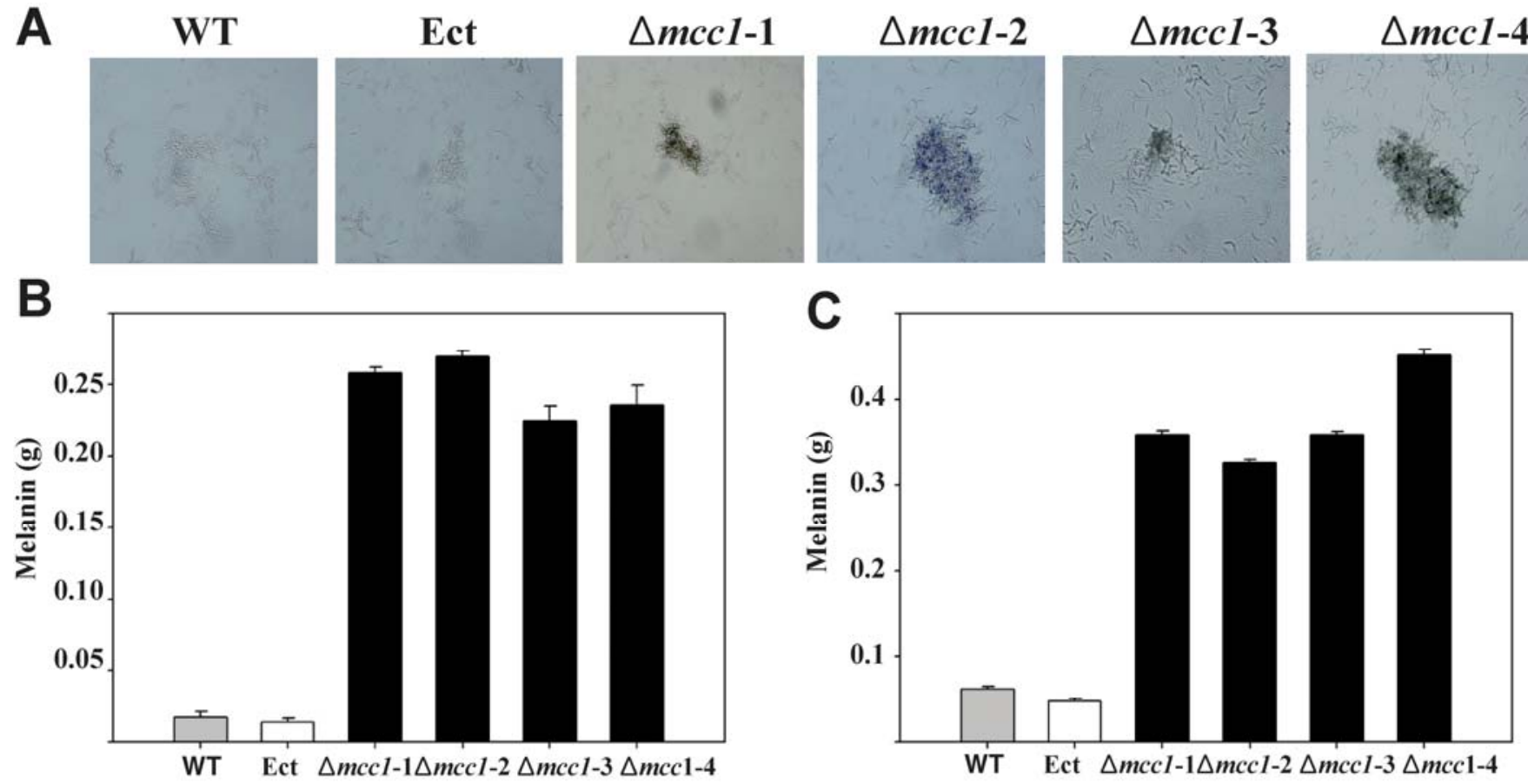

Fig. 3. Assays for melanin production. A, Yeast-sucrose broth (YSB) cultures of the wild type (WT), an ectopic transformant (Ect), and four independent $M C C 1$ mutants ( $\Delta m c c 1-1, \Delta m c c 1-2, \Delta m c c 1-3$, and $\Delta m c c 1-4)$ were photographed after incubation for 30 days. Black pigmentation was observed in $M C C 1$ gene-deletion mutants. B, Measurements of melanin production in the WT, Ect, and four $M C C 1$ gene-deletion mutants grown on yeast-sucrose agar for 30 days. Data from three independent replicates were analyzed to calculate means and standard errors, which are indicated by thin lines above the bars. C, Melanin production measurement in the WT, Ect, and four MCC1 gene-deletion mutants grown on YSB for 30 days. Data from three independent replicates were analyzed to calculate means and standard errors, indicated by thin lines above the bars. 
verified in microscopic images (Fig. 3A). Our results clearly indicate that the $M C C 1$ gene in $M$. graminicola is associated negatively with melanin biosynthesis.

\section{MCC1 gene deletion resulted}

in defects in filamentous growth.

Interestingly, we noticed significant defects of filamentous growth in the four independent $\Delta m c c 1$ mutants. Observation under the dissecting microscope clearly revealed that the full development of filamentous hyphae in $\Delta m c c 1$ mutants was lacking compared with the wild-type or ectopic controls. The wild-type and ectopic controls had long, well-developed hyphae extending to the margin of the colonies, whereas four $\Delta m c c l$ mutants produced short, aggregated hyphae and very restricted vegetative growth (Fig. 4A). These phenotypic characteristics were independent of growth temperature (Fig. 4A).

To confirm our observation, we tested germination morphology from single, isolated spores of the wild type, ectopic control, and four independent $\Delta m c c l$ mutants. After the same time period under identical environmental conditions, we observed consistent vegetative germination defects in all four $\mathrm{MCCl}$ gene-deletion mutants compared with the wild type or ectopic controls (Fig. 4B). As before, the wild type and ectopic controls produced elongated filaments. In contrast, all four $\mathrm{MCCl}$ gene-deletion mutants produced compact, small filaments with limited development (Fig. 4B). These vegetative germination defects in $\Delta m c c 1$ mutants appeared consistently in every single cell (more than 200) examined on water agar (WA) (data not shown). Measurement of vegetative growth also demonstrated that growth of $\Delta m c c l$ mutants was reduced $60 \%$ on WA compared with that of the wild type (Fig. 4C). Our results indicate that MCC1 plays a critical role in vegetative filamentous growth of $M$. graminicola.

\section{MCC1 gene deletion resulted in hyphal swellings.}

In contrast to the wild type and ectopic controls, the $\mathrm{MCCl}$ gene-deletion mutants ( $\Delta m c c 1-1, \Delta m c c l-2, \Delta m c c 1-3$, and $\Delta$ mccl-4) developed distinguishable hyphal swelling pheno-
A
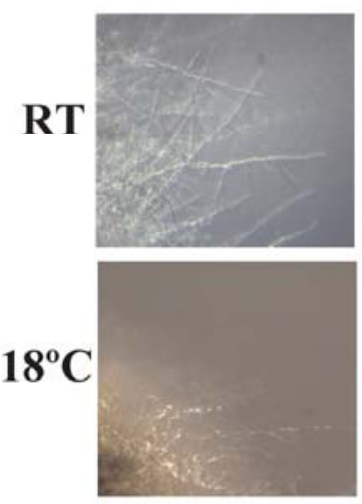

B

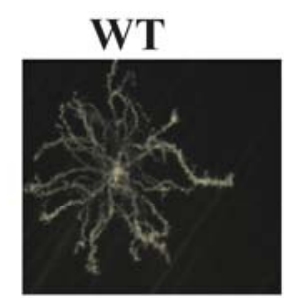

Ect
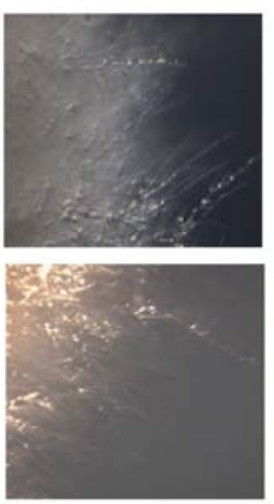

\section{Ect}

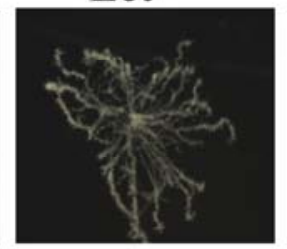

$\Delta m c c 1-1$
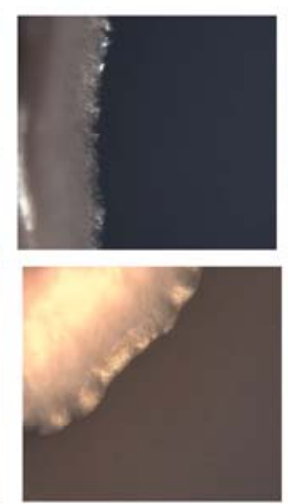

$\Delta m c c 1-1$

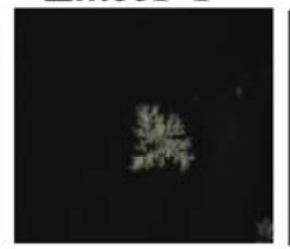

$\Delta m c c 1-2$
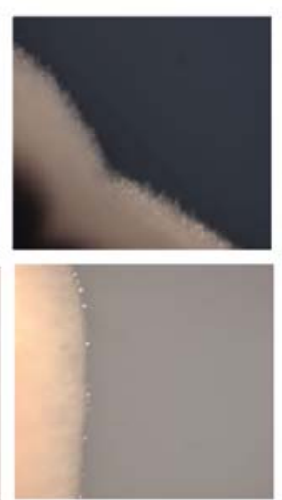

$\triangle m c c 1-2$

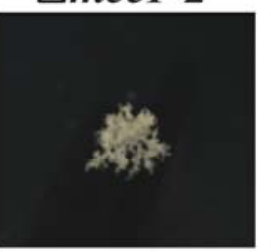

$\triangle m c c 1-3$
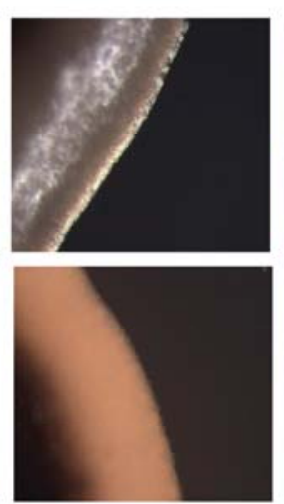

$\Delta m c c 1-3$

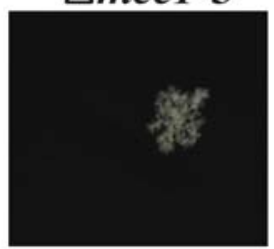

$\triangle m c c 1-4$
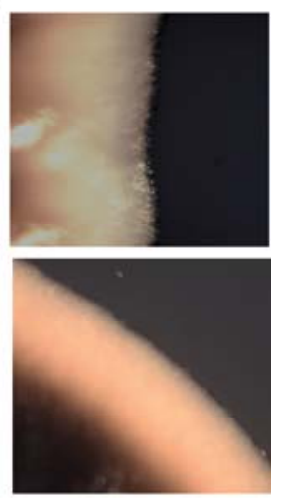

$\triangle m c c 1-4$

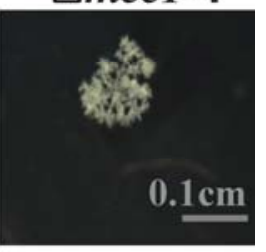

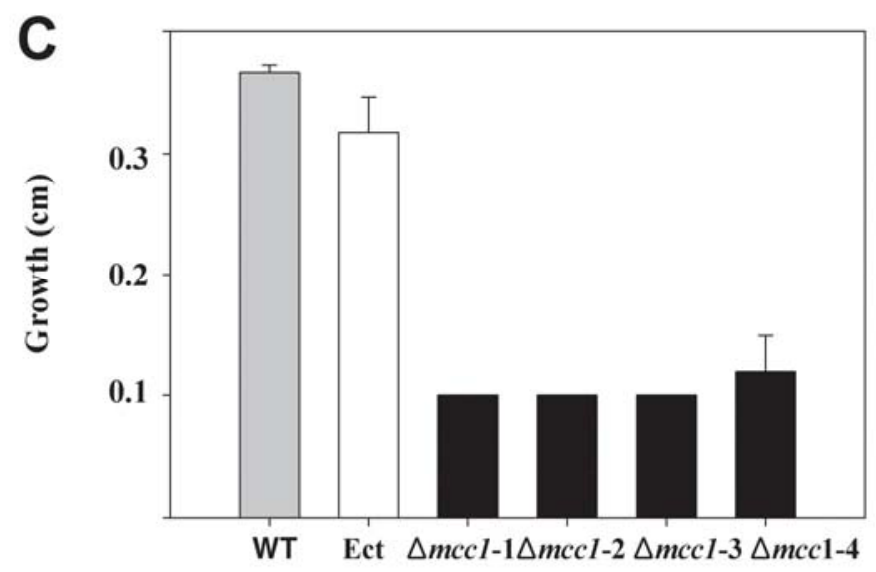

Fig. 4. Filamentous growth assays. A, Filamentous growth was photographed with a dissecting microscope. All strains were grown on potato dextrose agar (PDA) for 20 days at room temperature or at $18^{\circ} \mathrm{C}$ (indicated on the left). In the wild type (WT) and ectopic transformant (Ect) controls, invasive filamentous growth was present. However, $M C C 1$ mutants ( $\Delta m c c 1-1, \Delta m c c 1-2, \Delta m c c 1-3$, and $\Delta m c c 1-4)$ were defective in filamentous growth, regardless of temperature. $\mathbf{B}$, Defects in germination from a single cell in $M C C 1$ mutants. Single cells of all strains were isolated and spread on water agar. After 20 days of incubation at room temperature, germination patterns were photographed with a dissecting microscope. C, Germination growth in B was measured and presented as a bar graph. Results are means of three biological replications. Standard errors are indicated by thin lines above the bars; those for mutants $\Delta m c c 1-1, \Delta m c c 1-2$ and $\Delta m c c 1-3$ are too small to be visible at this scale. 
types when grown in liquid media (Fig. 5). These phenotypes in $\Delta m c c l$ mutants became apparent in the late growth stages (data not shown). The wild-type and ectopic control strains were capable of maintaining a filamentous growth pattern and similar size of cells even during the late stages, whereas $\Delta m c c 1$ mutants could not. Compared with the wild type and ectopic controls, all of the $M C C l$ gene-deletion mutants had consistent, abnormal balloon-shaped hyphae, which did not sustain a filamentous growth pattern after a certain time point (Fig. 5). Our results provide evidence that $M C C l$ in $M$. graminicola is involved in suppressing hyphal swelling or maintaining hyphal growth in late-stage cells.

\section{Disruption of $M C C 1$ increases stress tolerance to reactive oxygen species.}

Because the gene encoding c-type cyclin in $F$. graminearum is known to be involved in stress responses (Zhou et al. 2010), we assayed the effect of reactive oxygen species (ROS) on $\Delta m c c 1$ mutants as described previously (Choi and $\mathrm{Xu} 2010$ ). When grown on WA plates supplemented with $400 \mathrm{mM}$ concentrations of $\mathrm{H}_{2} \mathrm{O}_{2}$, growth of the wild-type and ectopic control strains was negligible and they did not survive (Fig. 6). However, all MCCl gene-deletion mutants survived on WA containing $400 \mathrm{mM} \mathrm{H}_{2} \mathrm{O}_{2}$, indicating that they had an increased tolerance to ROS. We also measured and quantified the relative growth of each strain by dividing its growth on media containing $\mathrm{H}_{2} \mathrm{O}_{2}$ by that on control media. The $\Delta m c c 1$ mutants had approximately 5- to 10- fold increases in growth on media containing $\mathrm{H}_{2} \mathrm{O}_{2}$ compared with the wild-type or ectopic control strains. These results support the hypothesis that $M C C 1$ in $M$. graminicola is associated with ROS stress-response signaling in a negative manner.

\section{MCC1 disruption significantly reduces pathogenicity.}

To determine the effect of $M C C 1$ deletion on virulence of $M$. graminicola, two infection assays were performed on the highly susceptible wheat 'Taichung 29'. Wheat seedlings at the three- to five-leaf stage were inoculated separately with $10^{7}$ spores of each strain. Both stem and spray inoculations were performed and plants were incubated until the appearance of STB disease symptoms, such as pycnidia indicated by black spots within tan lesions. We obtained consistent results from all four independent $M C C 1$ mutants; STB caused by $M C C 1$

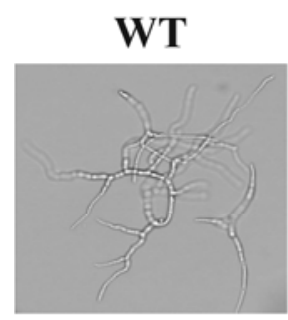

$\Delta m c c 1-2$
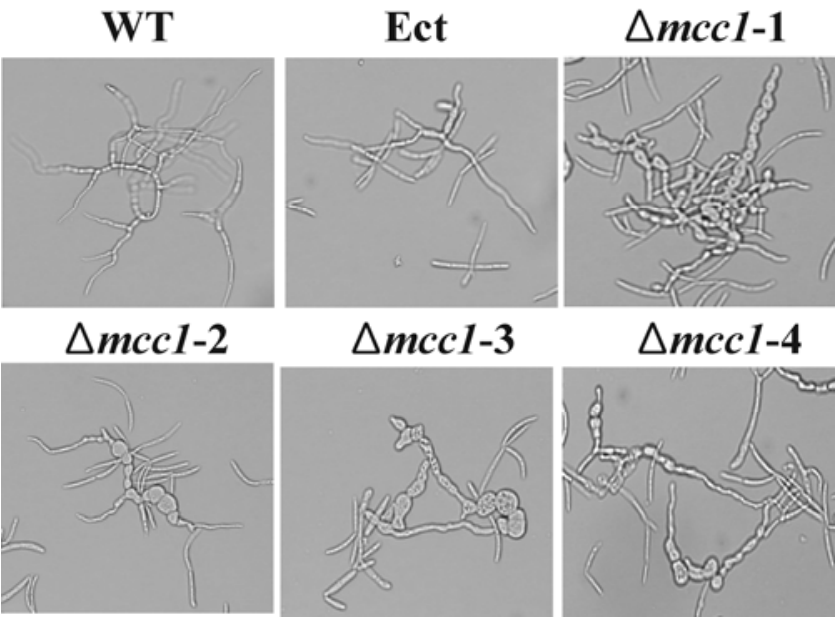

Fig. 5. Hyphal swelling phenotype associated with $M C C 1$ mutation in Mycosphaerella graminicola. Each strain was inoculated in defined liquid medium with the same amount of conidia $\left(1 \times 10^{5}\right.$ fungal cells $\left.\mathrm{ml}^{-1}\right)$. After 30 days of incubation at $18^{\circ} \mathrm{C}$, photos were taken under the microscope. WT: wild type; Ect: ectopic transformant control; $\Delta m c c 1-1, \Delta m c c 1-2$, $\Delta m c c 1-3$, and $\Delta m c c 1-4$ : four independent $M C C 1$ mutants. mutants was significantly limited, compared with that caused by the wild-type and ectopic control strains (Fig. 7A and B). Wild-type and ectopic control strains were capable of successfully colonizing the leaves of Taichung 29 wheat, showing the typical black spots of pycnidia, and these symptoms were spread across the wheat leaves. However, we observed significant reductions in the progression of STB in four $\Delta m c c 1$ strains (Fig. 7A and B). STB symptoms from $M C C l$ gene-deletion strains were barely detectable or even nondetectable, and these defects in pathogenicity were consistent, regardless of inoculation method (Fig. 7A and B). Taken together, we concluded that the MCC1 gene in M. graminicola is associated with pathogenicity and virulence on wheat.

\section{DISCUSSION}

Cyclins are a group of well-conserved proteins which are crucial for the progressions of cell cycles and are important determinants of cell fates (Fung and Poon 2005). Cyclins act by forming complexes with $\mathrm{CDK}$, and these cyclin-CDK complexes direct the activation of cell cycle progressions. Several different types of cyclins and matching CDK are known, tightly regulating different phases of cell cycle transitions. To do so, the concentrations of cyclins oscillate at the protein level throughout the cell cycle which, in turn, leads to cell cycle progressions (Ekholm and Reed 2000; Morgan 1995; Sherr 1993).

Of the different cyclins, c-type cyclin has distinct features because it is a component of RNA polymerase II involved in transcriptional regulation (Liao et al. 1995). The carboxy-terminal domain (CTD) of RNA polymerase II has been implicated in transcriptional regulation (Dahmus 1996). In S. cerevisiae, c-type cyclin Srb11p (Ume3p) and a CDK, Srb10p complex, is physically associated with the CTD of RNA polymerase II. The Srb11p-Srb10p pair phosphorylates CTD accordingly, allowing transcription of many downstream genes to be activated or repressed (Liao et al. 1995). In addition, unlike the other cyclins, protein concentration of c-type cyclin does not fluctuate throughout the cell cycle (Cooper et al. 1997; Molz and Beach 1993).

Thus far, most of the information obtained on c-type cyclin originated from yeast, Drosophila spp., and human oncogenes. However, recent research has begun to reveal interesting functional roles of c-type cyclin in filamentous fungi. Emerging evidence from Fusarium spp. suggested that c-type cyclins

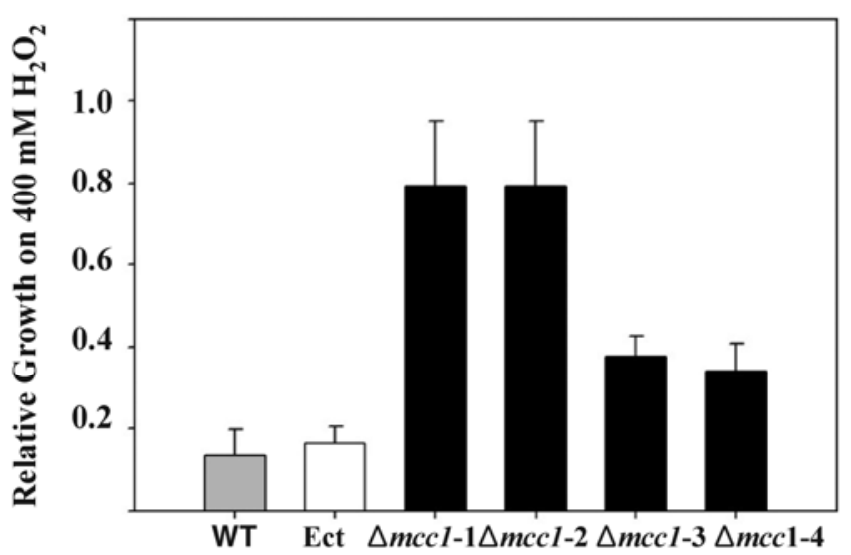

Fig. 6. Increased $\mathrm{H}_{2} \mathrm{O}_{2}$ tolerance in $\Delta m c c 1$ mutants. Relative growth of each strain was calculated by comparing its radial growth on potato dextrose agar plates with 400-mM concentrations of $\mathrm{H}_{2} \mathrm{O}_{2}$ to those on plates with no $\mathrm{H}_{2} \mathrm{O}_{2}$. Wild type (WT) and ectopic transformant (Ect) control strains could not survive on $400 \mathrm{mM} \mathrm{H}_{2} \mathrm{O}_{2}$, whereas all of the $\Delta m c c 1$ mutants ( $\Delta m c c 1-1$, $\Delta m c c 1-2, \Delta m c c 1-3$, and $\Delta m c c 1-4)$ were able to survive and grow. 
have fundamental roles in fungal development, production of secondary metabolites, and pathogenicity (Shim and Woloshuk 2001; Zhou et al. 2010). Nevertheless, we have limited knowledge about c-type cyclin, because only two c-type cyclin genes, FCCl in F. verticillioides and CIDI in F. graminearum, have been characterized in filamentous fungi.

In this study, we attempted to characterize the c-type cyclin gene, $M C C 1$, in $M$. graminicola. As with other c-type cyclin genes in different species, $M C C 1$ gene-deletion mutants were not lethal. Moreover, we identified multiple phenotypes in MCC1 mutants, indicating that $M C C 1$ is involved in multiple signaling in $M$. graminicola. Four independent $\Delta m c c l$ mutants were generated and exhibited consistent phenotypes, suggesting that $M C C l$ is truly associated with all of the phenotypes observed in this study.

Deletion of the $M C C 1$ gene led to phenotypes that were very similar to those of the FCC1 and CIDl gene deletions (Shim and Woloshuk 2001; Zhou et al. 2010). Phylogenetically, $M$. graminicola is placed in the order Capnodiales (Schoch et al. 2006; Torriani et al. 2008), distantly related to the order Hypocreales, in which Fusarium spp. are placed (Goodwin 2004; Schoch et al. 2006). In spite of the evolutionary divergence between the Hypocreales and Capnodiales, we observed very similar consequences of c-type cyclin gene deletions. Moreover, amino acid sequence analysis of $M C C 1, F C C 1$, and CIDl demonstrated that $78 \%$ of Mcc1p amino acids were either identical or similar to those of Fcc1p and Cid1p. Therefore, we propose that functional roles of c-type cyclin genes in filamentous fungi are well conserved across many divergent fungal species.

Deletion of the $M C C 1$ gene resulted in significant reduction in growth, which also was reported in Fusarium spp. In Fusarium spp., deletion of the c-type cyclin gene led to an approximately 25\% reduction in growth (Shim and Woloshuk 2001; Zhou et al. 2010), whereas approximately 50\% reduction occurred in M. graminicola (Fig. 1B). When we searched for putative homologs of c-type cyclin genes, we found two and three copies in the genomes of $F$. verticillioides and $F$. graminearum, respectively. However, only one putative homolog of a c-type cyclin gene was identified in the genome of $M$. graminicola (data not shown). Therefore, the absence of additional redundant c-type cyclin genes in the genome of $M$. graminicola may explain the more severe growth defects in MCC1 deletion mutants compared with those in knock-out strains of homologous genes of Fusarium spp.

We also observed defects in filamentous growth in $\Delta m c c 1$ mutants, with a phenotype of short and condensed germination tubes (Fig. 4). The CID1 mutant in F. graminearum also
A

WT

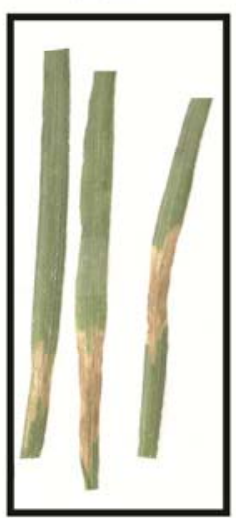

B

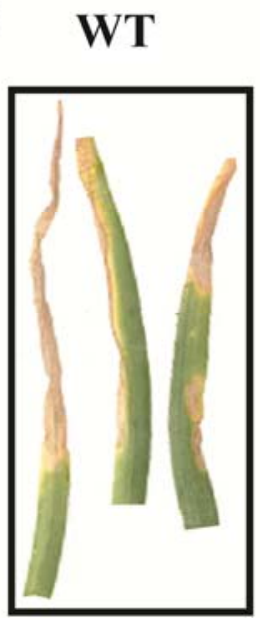

Ect

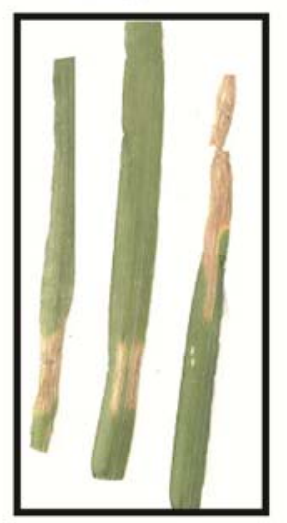

Ect

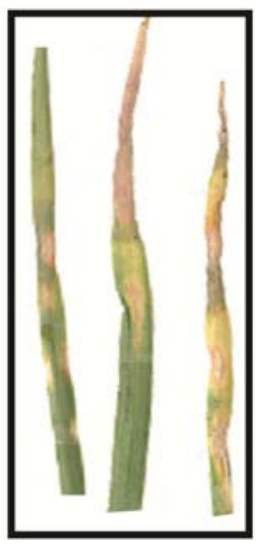

$\triangle m c c 1-1$

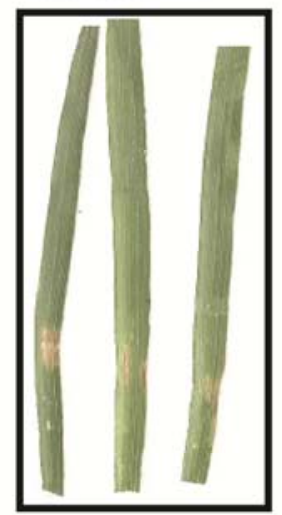

$\triangle m c c 1-1$

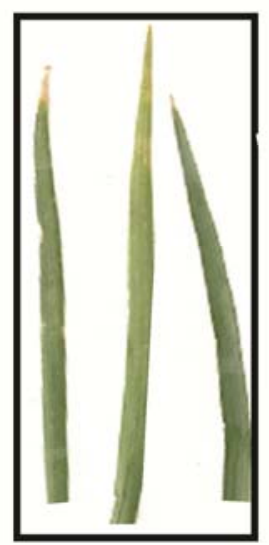

$\triangle m c c 1-2$

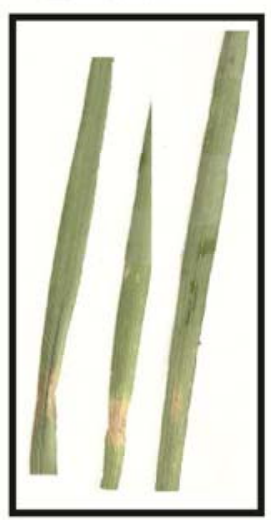

$\triangle m c c 1-2$

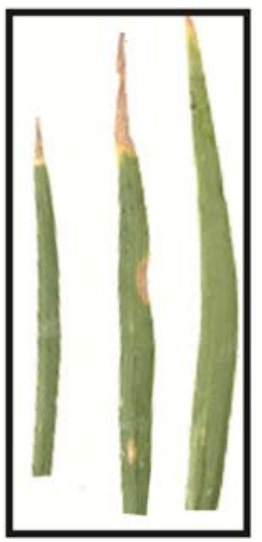

$\triangle m c c 1-3$

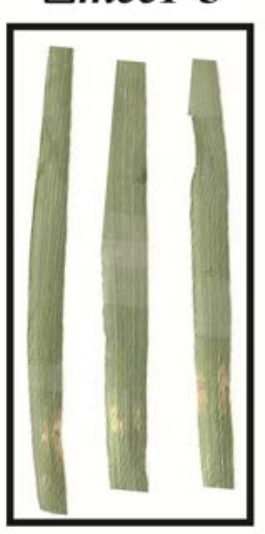

$\triangle m c c 1-3$

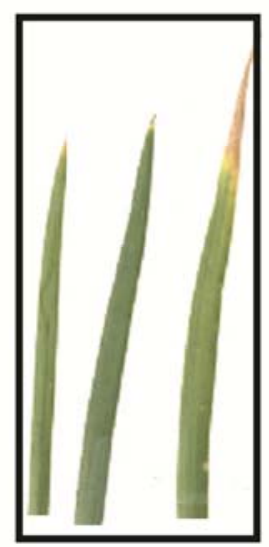

$\triangle m c c 1-4$

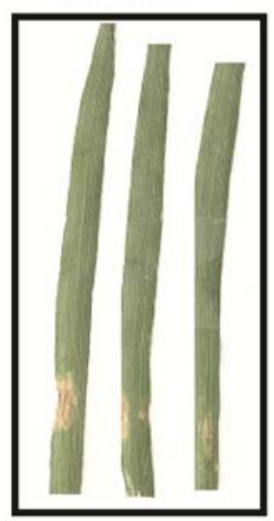

$\triangle m c c 1-4$

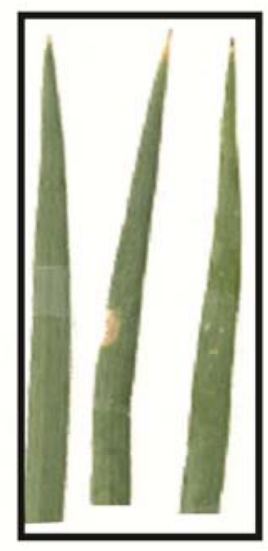

Fig. 7. Effect of $M C C 1$ gene deletion on pathogenicity. A, Stem infection assays. Sheaths of the susceptible wheat 'Taichung 29' were punctured with a sterile needle and infected with $3 \times 10^{7}$ spores of the wild type (WT), an ectopic transformant (Ect) control strain, and four independent $M C C 1$ gene-deletion mutants ( $\Delta m c c 1-1, \Delta m c c 1-2, \Delta m c c 1-3$, and $\Delta m c c 1-4)$. Infected wheat plants were incubated in a Purdue University greenhouse facility for 21 days. Stem infection assays were performed with at least three biological replications for each fungal isolate. Symptoms are visible only near the sites of inoculation. B, Spray infection assays. In all, $10^{8}$ spores of WT, Ect, and four independent $M C C 1$ gene-deletion mutants $(\Delta m c c 1-1, \Delta m c c 1-2, \Delta m c c 1-3$, and $\Delta m c c 1-4)$ were spray inoculated onto seedling leaves of highly susceptible Taichung 29 wheat. After inoculation, plants were placed in the dark for $48 \mathrm{~h}$ under high humidity. Subsequently, the infected wheat plants were incubated in a Purdue University greenhouse facility with cycles of $12 \mathrm{~h}$ of light and $12 \mathrm{~h}$ of darkness for 21 days. Spray infection assays were performed with at least three biological replications for each fungal isolate. 
showed a delay in germ tube elongation (Zhou et al. 2010). Therefore, c-type cyclin in fungi is likely responsible for filamentous growth or hypal elongation, which are likely to involve similar processes. However, because c-type cyclin null mutants are still able to grow filamentously, even though impaired, there should be one or more additional and indispensable signals for filamentous growth. Presumably, c-type cyclin may be involved in full development of filamentous growth in the late phases of elongating germ tubes. Further study will be necessary to understand the functional role or roles of c-type cyclin in filamentous growth.

Interestingly, we observed hyphal swelling phenotypes in $\Delta m c c 1$ mutants (Fig. 5). Previously, Choi and Shim (2008) showed that knock-out mutation of the protein phosphatase gene $C P P 1$ resulted in hyphal swelling similar to that in $\triangle m c c 1$ mutants, and $C P P 1$ was identified initially as a downstream gene of c-type cyclin FCC1 (Pirttilä et al. 2004; Shim and Woloshuk 2001). Thus, it is likely that MCC1 is associated with hyphal swelling via a Cpplp-homologous protein in $M$. graminicola. Although hyphal swelling was not reported in the other studies on the c-type cyclin gene, we believe that hyphal swelling phenotypes probably occur in the other cyclin gene mutants, considering the high conservation of functional roles of c-type cyclins. Additionally, a previous report on deletion mutants of $M g S l t 2$, encoding a mitogen-activated protein (MAP) kinase in $M$. graminicola, also showed large swollen cells (Mehrabi et al. 2006a). Further study is required to reveal the molecular relationships between $M C C 1$ and $M g S l t 2$.

We also found that $M C C l$ deletion led to significant decreases in development of aerial mycelium (Fig. 2). Previous reports have documented that fungal c-type cyclin influences conidiation positively (Shim and Woloshuk 2001; Zhou et al. 2010). However, in this study, MCC1 was instead positively associated with development of aerial mycelium. Because fungal conidia develop on the tips of aerial hyphae, it is likely that the conidial defects observed in the FCCI and CIDI gene-deletion mutants also may have originated from lack of aerial mycelium rather than a defect in conidia formation itself. In $A$. nidulans, three genes ( $b r l A, a b a A$, and wetA) are involved in key regulatory pathways for conidiation, controlling conidiaspecific gene expression or activation in a specific sequence (Adams et al. 1998). Of these, brlA is responsible for the early development of conidiophore vesicles and regulating transcript accumulation of $a b a A$ and wetA (Boylan et al. 1987; Clutterbuck 1969). Because $M C C 1$ deletions affect formation of aerial mycelium, which should take place before the development of conidiophore vesicles, we propose that c-type cyclin signaling in filamentous fungi might be upstream of $b r l A$, influencing all of the regulatory genes controlling conidia development.

Another possibility might be found in the relationship between c-type cyclin and hydrophobins. For many fungal species, secreted proteins known as hydrophobins enable aerial hyphae to develop by decreasing the water surface tension (Wösten and Willey 2000). Thus, the relationship between the hydrophobins and c-type cyclin also should be determined in the future.

We noticed that $\Delta m c c 1$ mutants had increased melanin production (Figs. 1A and 3). Previously, c-type cyclin genes in Fusarium spp. were associated with mycotoxins or secondary metabolites (Shim and Woloshuk 2001; Zhou et al. 2010). In M. graminicola, MCCl directs production of another secondary metabolite, melanin, indicating functional conservation of c-type cyclins in the secondary metabolism of fungi. Furthermore, we could not eliminate the possibility that $M C C 1$ also might be implicated in production of mycotoxins or secondary metabolites as yet unidentified in M. graminicola. Evidence from Fusarium spp. tells us that the same c-type cyclin gene is implicated in production of several secondary metabolites, including mycotoxins as well as pigments (Zhou et al. 2010). Based on that, we suspected that it also might be true in $M$. graminicola.

Another phenotype of $\Delta m c c l$ mutants was their increased stress tolerance, indicating that $M C C 1$ might function as a repressor of stress responses (Fig. 6). In S. cerevisiae, the c-type cyclin protein Ume $3 p$ functions as a repressor of stress-response gene transcription. To escape from this repression, Ume $3 p$ must be destroyed via ubiquitin-mediated proteolysis in response to environmental stresses (Cohen et al. 2003; Cooper et al. 1997). In this regard, it is conceivable that $M C C 1$ gene deletion activates many downstream stress-response genes which, in turn, lead to more stress tolerance. Interestingly, the CID1 mutant in F. graminearum was defective in stress responses (Zhou et al. 2010). Therefore, c-type cyclin genes in $F$. graminearum and $M$. graminicola seem to have opposite roles in stress responses, although most of the phenotypes observed indicate well-conserved functional roles. In addition, several reports demonstrated that fungal melanin has antioxidant properties (Jacobson and Tinnell 1993; Shcherba et al. 2000). It remains to be determined whether increased melanin biosynthesis in $\Delta m c c 1$ mutants is directly related to ROS tolerance.

We also observed significant reductions in pathogenicity in MCC1 gene-deletion mutants, regardless of inoculation method (Fig. 7A and B). This is consistent with the previous results with the CID1 mutant, suggesting c-type cyclin involvement in pathogenicity in filamentous fungi (Zhou et al. 2010). Therefore, it will be interesting to elucidate signal-transduction convergence or the relationship of fungal c-type cyclin genes with well-known signaling pathways for pathogenicity such as the MAP kinase or cAMP signaling pathways. It also is noteworthy that there may be a connection between defects in filamentous growth in $M C C 1$ deletion mutants and pathogenicity.

As with ume3 in $S$. cerevisiae, c-type cyclin in filamentous fungi supposedly has regulatory roles in gene transcription, including multiple signaling genes to generate the variety of phenotypes observed in this study. However, we still have limited knowledge of genes that act downstream of c-type cyclin. So far, only genes downstream of FCCl have been studied extensively via microarray or cDNA-subtraction-library techniques (Pirttilä et al. 2004; Shim and Woloshuk 2001). To better understand $\mathrm{MCCl}$ regulation on gene transcription, it will be important to investigate genome-wide mRNA transcription with microarrays or other approaches. Furthermore, MCC1 undoubtedly regulates the phenotypes observed in this study via CDK, similar to the CDK protein Fck1 in F. verticillioides, which has a pleiotropic effect on several morphological processes through its interactions with Fcc1 (Bluhm and Woloshuk 2006). Further study with $M$. graminicola CDK will be necessary to unravel $M C C 1$-mediated signaling pathways.

In summary, we characterized $M C C 1$, a c-type cyclin gene in $M$. graminicola, and demonstrated that it plays important roles in multiple phenotypes: development of aerial mycelium, filamentous growth, hyphal swellings, melanin biosynthesis, stress tolerance response, and pathogenicity. Further research will be necessary to elucidate the exact mechanism of each type of ctype cyclin-mediated signaling pathway. Because many of the phenotypes described in this study are consistent with those in previous reports on c-type cyclin mutants in Fusarium spp., we concluded that the functional roles of $\mathrm{MCC}$ 1-homologous genes are well conserved across species in the fungal kingdom.

\section{MATERIALS AND METHODS}

\section{Fungal strains and culture media.}

Highly virulent $M$. graminicola strain IPO323 was used as the wild type throughout this study and was the subject of gene 
disruptions. All of the strains used or generated in this study were stored at $-80^{\circ} \mathrm{C}$ after desiccation on strips of Whatman filter paper (Whatman Inc., Piscataway, NJ, U.S.A.) overnight in a lyophilizer. Cultures grown in YSB (10 g each of sucrose and yeast extract [Difco Laboratories, Detroit] per liter of distilled water) were used for genomic DNA extraction or ATMT. For phenotyping, we used various agar media: YSA (YSB with $15 \mathrm{~g}$ of agar per liter); PDA (4\%; Difco Laboratories); and WA (15 $\mathrm{g}$ of agar per liter). For hyphal swelling studies, defined liquid medium consisted of $22 \mathrm{mM} \mathrm{KH}_{2} \mathrm{PO}_{4}, 2.5 \mathrm{mM} \mathrm{MgSO}$, $85 \mathrm{mM} \mathrm{NaCl}, 10 \mathrm{mM} \mathrm{NH}_{4} \mathrm{NO}_{3}$, and $117 \mathrm{mM}$ sucrose.

\section{Nucleic acid isolation and manipulation.}

All plasmid DNAs used in this study were prepared with the QIAprep Spin Miniprep Kit (Qiagen, Valencia, CA, U.S.A.). The $M C C 1$ gene knock-out construct, DMC1, was made via double-joint PCR as described previously (Yu et al. 2004). All primers used for PCR are listed in Supplementary Table 1. Primer pairs MCC1-dis-A and MCC1-dis-B and MCC1-dis-C and MCC1-dis-D were used to amplify flanking regions of approximately $1 \mathrm{~kb}$ around the $M C C l$ gene from $M$. graminicola genomic DNA. Simultaneously, an $\mathrm{HPH}$ gene was amplified from plasmid vector pBHT2 (Mullins et al. 2001) with HPH-F and HPH-R primers using AccuSure DNA Polymerase, which has proof-reading activity (Bioline, Taunton, MA, U.S.A.). Subsequently, the three amplicons were mixed in a single tube in a 1:3:1 (5' fragment: marker: $3^{\prime}$ fragment) molar ratio and joined by PCR without using primers as described previously (Yu et al. 2004). Finally, nested primers MCC1nest-F and MCC1-nest-R were used to amplify the $3.5-\mathrm{kb}$ DMC1 construct. Primers MCC1-nest-F and MCC1-nest-R contain recognition sites for the restriction enzymes EcoRI and $K p n I$, respectively. With these enzyme sites, the DMC1 construct was cloned into pBHT2 vector (Mullins et al. 2001) for ATMT. The created vector, designated pMC1, was transformed into competent cells of Agrobacterium strain LBA1100 by electroporation following the standard protocols for further use of ATMT.

\section{ATMT.}

An Agrobacterium strain containing the $M C C l$ knock-out ATMT construct, pMC1, was used for transformation into $M$. graminicola. Cells for gene disruption were grown in YSB at $18^{\circ} \mathrm{C}$ for 2 weeks in an orbital incubated shaker. ATMT was carried out using fungal spores adjusted to $1 \times 10^{7}$ spores $/ \mathrm{ml}$ by essentially following the protocol described previously (Zwiers and de Waard 2001). PDA containing $100 \mu \mathrm{M}$ hygromycin and $200 \mu \mathrm{M}$ cefotaxime was used for the selective medium. After 2 to 3 weeks, hygromycin-resistant colonies appeared on selective medium and every isolated colony was transferred for subsequent subculture on selective medium for further verification of DMC1 incorporation into the genome.

\section{Single-spore isolations and germination study.}

We isolated single spores of $M$. graminicola by spreading extremely low concentrations of fungal spores (fewer than 50 spores/plate) on WA. Hemacytometer counts were used for adjustments of fungal spore concentration and serial dilutions were performed to obtain the expected concentration of fungal spores of each strain. After incubating at room temperature for 20 days, shapes of the germination were investigated. Three or more biological replications were performed.

\section{Microscopy.}

Microscopic observations were made with either a dissecting or compound microscope (Olympus America Inc., Melville, NY, U.S.A.). For images of aerial mycelia and filamentous growth, we used dissecting microscope model SZX12 and Qcapture software. Analysis of hyphal growth phenotypes was performed using DP controller software and an Olympus BX41 microscope equipped with a DP70 camera.

\section{Melanin assays.}

Melanin was extracted from total mycelium grown on YSA and YSB at room temperature for 30 days. Fungal strains grown in $5 \mathrm{ml}$ of YSB in 14-ml polypropylene tubes (BD Biosciences, San Jose, CA, U.S.A.) were used for melanin analysis. Melanin was extracted following the protocol described previously (Babitskaya et al. 2000). Briefly, melanin was extracted with $2 \% \mathrm{NaOH}$ at $100^{\circ} \mathrm{C}$ for $2 \mathrm{~h}$ in a water bath. The cooled extracts were acidified to $\mathrm{pH} 2.0$ with $\mathrm{HCl}$, precipitating the melanin pigments. Subsequently, extracts were centrifuged to separate melanin from extract solution and the resulting pellets were dissolved in $2 \% \mathrm{NaOH}$. The absorbance of the extract was measured spectrophotometrically at a wave length of $459 \mathrm{~nm}$ and the amount of melanin was determined by a calibration curve plotted from the results of a correlation between melanin dry weight and absorbance. All assays were replicated at least three times.

\section{Stress tolerance assays.}

To assay the responses to oxidative stress, identical $0.2-\mathrm{cm}-$ diameter agar blocks excised from actively growing cultures were inoculated onto PDA (4\%; Difco Laboratories) plates containing 100,200 , or $400 \mathrm{mM} \mathrm{H}_{2} \mathrm{O}_{2}$. Radial growth was analyzed after incubation at room temperature for 21 days. All assays were repeated at least three times.

\section{Virulence assays.}

The susceptible wheat line Taichung 29 was inoculated by either stem or spray inoculations. Briefly, we grew Taichung 29 in a Purdue University greenhouse facility until the wheat seedlings reached the three- to five-leaf stage. Fungal inoculum was prepared from 2-week-old cultures of M. graminicola grown in YSB at room temperature on an orbital shaker adjusted to $120 \mathrm{rpm}$. For the stem-infection assay, wheat stems were punctured with a sterile needle and $3 \times 10^{7}$ spores of each strain were inoculated into the wound. For spray inoculations, we followed the protocol described previously (Mehrabi et al. $2006 \mathrm{a}$ and $\mathrm{b}$ ). Wheat at the above-mentioned stage was inoculated by spraying $10^{7}$ spores suspended in water per pot. After inoculation, wheat pots were placed in the dark for $48 \mathrm{~h}$ while tightly maintaining full humidity. Subsequently, infected wheat plants were incubated in cycles of $12 \mathrm{~h}$ of light and $12 \mathrm{~h}$ of darkness. After 21 days, when unmistakable STB disease symptoms of clear black pycnidia spots appeared, we analyzed the extent of the infections. All assays were repeated at least three times.

\section{ACKNOWLEDGMENTS}

We thank L. Dunkle and C. Woloshuk for critical reading of the manuscript; and L. Dunkle, T. Mengiste, and R. Latin for use of microscopes and experimental equipment. This work was supported by United States Department of Agriculture-Agricultural Research Service (USDA-ARS) CRIS project 3602-22000-015-00D and by a USDA-ARS AdministratorFunded postdoctoral position to Y.-E. Choi.

\section{LITERATURE CITED}

Adams, T. H., Wieser, J. K., and Yu, J-H. 1998. Asexual sporulation in Aspergillus nidulans. Microbiol. Mol. Biol. Rev. 62:35-54.

Babitskaya, V. G., Shcherba, V. V., Filimonova, T. V., and Grigorchuk, E. A. 2000. Melanin pigments from the fungi Paecilomyces variotii and Aspergillus carbonarius. Appl. Biochem. Microbiol. 36:128-133. 
Bluhm, B. H., and Woloshuk, C. P. 2006. Fck1, a c-type cyclin-dependent kinase interacts with Fcc1 to regulate development and secondary metabolism in Fusarium verticillioides. Fungal Genet. Biol. 43:146-154.

Boylan, M. T., Mirabito, P. M., Willett, C. E., Zimmerman, C. R., and Timberlake, W. E. 1987. Isolation and physical characterization of three essential conidiation genes from Aspergillus nidulans. Mol. Cell. Biol. 7:3113-3118

Choi, Y.-E., and Shim, W.-B. 2008. Functional characterization of Fusarium verticillioides $C P P 1$, a gene encoding putative protein phosphatase 2A catalytic subunit. Microbiology 154:326-336.

Choi, Y.-E., and Xu, J.-R. 2010. The cAMP signaling pathway in Fusarium verticillioides is important for conidiation, plant infection, and stress responses but not fumonisin production. Mol. Plant-Microbe Interact. 23:522-533.

Clutterbuck, A. J. 1969. A mutational analysis of conidial development in Aspergillus nidulans. Genetics 63:317-327.

Cohen, T. J., Lee, K., Rutkowski, L. H., and Strich, R. 2003. Ask10p mediates the oxidative stress-induced destruction of the Saccharomyces cerevisiae c-type cyclin Ume3p/Srb11p. Eukaryot. Cell 2:962-970.

Cooper, K., Mallory, M. J., Smith, J. B., and Strich, R. 1997. Stress and developmental regulation of the yeast C-type cyclin Ume3p (Srb11p/Ssn8p). EMBO (Eur. Mol. Biol. Organ.) J. 16:4665-4675.

Dahmus, M. E. 1996. Reversible phosphorylation of the C-terminal domain of RNA polymerase II. J. Biol. Chem. 271:19009-19012.

Ekholm, S. V., and Reed, S. I. 2000. Regulation of G1 cyclin-dependent kinases in the mammalian cell cycle. Curr. Opin. Cell Biol. 12:676-684.

Fung, T. K., and Poon, R. Y. C. 2005. A roller coaster ride with the mitotic cyclins. Semin. Cell. Dev. Biol. 16:335-342.

Goodwin, S. B. 2004. Minimum phylogenetic coverage: an additional criterion to guide the selection of microbial pathogens for initial genomics sequencing efforts. Phytopathology 94:800-804.

Jacobson, E. S., and Tinnell, S. B. 1993. Antioxidant function of fungal melanin. J. Bacteriol. 175:7102-7104.

Kuchin, S., Yeghiayan, P., and Carlson, M. 1995. Cyclin-dependent protein kinase and cyclin homologs SSN3 and SSN8 contribute to transcriptional control in yeast. Proc. Natl. Acad. Sci. U.S.A. 92:4006-4010.

Liao, S.-M., Zhang, J., Jeffery, D. A., Koleske, A. J., Thompson, C. M., Chao, D. M., Viljoen, M., van Vuuren, H. J. J., and Young, R. A. 1995. A kinase-cyclin pair in the RNA polymerase II holoenzyme. Nature 374:193-196.

Mehrabi, R., van der Lee, T., Waalwijk, C., and Kema, G. H. J. $2006 a$. MgSlt2, a cellular integrity MAP kinase gene of the fungal wheat pathogen Mycosphaerella graminicola, is dispensable for penetration but essential for invasive growth. Mol. Plant-Microbe Interact. 19:389-398

Mehrabi, R., Zwiers, L.-H., de Waard, M. A., and Kema, G. H. J. 2006b. MgHogl regulates dimorphism and pathogenicity in the fungal wheat pathogen Mycosphaerella graminicola. Mol. Plant-Microbe Interact. 19:1262-1269.
Molz, L., and Beach, D. 1993. Characterization of the fission yeast mcs 2 cyclin and its associated protein kinase activity. EMBO (Eur. Mol. Biol. Organ.) J. 12:1723-1732.

Morgan, D. O. 1995. Principles of CDK regulation. Nature 374:131-134.

Mullins, E. D., Chen, X., Romaine, P., Raina, R., Geiser, D. M., and Kang, S. 2001. Agrobacterium-mediated transformation of Fusarium oxysporum: an efficient tool for insertional mutagenesis and gene transfer. Phytopathology 91:173-180.

Pirttilä, A. M., McIntyre, L. M., Payne, G. A., and Woloshuk, C. P. 2004. Expression profile analysis of wild-type and $f c c l$ mutant strains of Fusarium verticillioides during fumonisin biosynthesis. Fungal Genet. Biol. 41:647-656.

Polley, R. W., and Thomas, M. R. 1991. Surveys of diseases of winter wheat in England and Wales 1976-88. Ann. Appl. Biol. 119:1-20.

Schoch, C. L., Shoemaker, R. A., Seifert, K. A., Hambleton, S., Spatafora, J. W., and Crous, P. W. 2006. A multigene phylogeny of the Dothideomycetes using four nuclear loci. Mycologia 98:1041-1052.

Shcherba, V. V., Babitskaya, V. G., Kurchenko, V. P., Ikonnikova, N. V., and Kukulyanskaya, T. A. 2000. Antioxidant properties of fungal melanin pigments. Appl. Biochem. Microbiol. 36:491-495.

Sherr, C. J. 1993. Mammalian G1 cyclins. Cell 73:059-1065.

Shim, W. B., and Woloshuk, C. P. 2001. Regulation of fumonisin B B $_{1}$ biosynthesis and conidiation in Fusarium verticillioides by a cyclin-like (C-Type) gene, FCC1. Appl. Environ. Microbiol. 67:1607-1612.

Torriani, S. F. F., Goodwin, S. B., Kema, G. H. J., Pangilinan, J. L., and McDonald, B. A. 2008. Intraspecific comparison and annotation of two complete mitochondrial genome sequences from the plant pathogenic fungus Mycosphaerella graminicola. Fungal Genet. Biol. 45:628-637.

Wösten, H. A. B., and Willey, J. M. 2000. Surface-active proteins enable microbial aerial hyphae to grow into the air. Microbiology 146:767773.

Yu, J.-H., Hamari, Z., Han, K.-H., Seo, J.-A., Reyes-Domínguez, Y., and Scazzocchio, C. 2004. Double-joint PCR: a PCR-based molecular tool for gene manipulations in filamentous fungi. Fungal Genet. Biol. 41:973-981.

Zhou, X., Heyer, C., Choi, Y.-E., Mehrabi, R., and Xu, J.-R. 2010. The CID1 cyclin C-like gene is important for plant infection in Fusarium graminearum. Fungal Genet. Biol. 47:143-151.

Zwiers, L.-H., and de Waard, M. A. 2001. Efficient Agrobacterium tumefaciens-mediated gene disruption in the phytopathogen Mycosphaerella graminicola. Curr. Genet. 39:388-393.

\section{AUTHOR-RECOMMENDED INTERNET RESOURCES}

Joint Genome Institute $M$. graminicola genomic database: genome.jgi-psf.org/Mycgr3/Mycgr3.home.html

SMART (Simple Modular Architecture Research Tool) database: smart.embl-heidelberg.de 